\title{
Design, synthesis and antidiabetic evaluation of oxazolone derivatives
}

\author{
G MARIAPPAN $^{\mathrm{a} *}$, B P SAHA ${ }^{\mathrm{a}}$, SRIPARNA DATTA ${ }^{\mathrm{a}}$, DEEPAK KUMAR ${ }^{\mathrm{a}}$ and P K HALDAR ${ }^{\mathrm{b}}$ \\ ${ }^{a}$ Department of Medicinal Chemistry, ${ }^{b}$ Department of Pharmacology, Himalayan Pharmacy Institute, \\ East Sikkim 737 136, India \\ e-mail: gmariappanhpi@yahoo.co.in
}

MS received 1 December 2010; revised 16 March 2011; accepted 18 March 2011

\begin{abstract}
A series of ten novel (2a-j) 4-arylidine 2-[4-methoxy phenyl] oxazol-5-one derivatives were synthesized and assayed in vivo to investigate their antidiabetic activities by streptozotocin-induced model in rat. These derivatives showed considerable biological efficacy when compared to rosiglitazone, a potent and wellknown antidiabetic agent as a reference drug. All the compounds were effective, amongst them 2d shows more prominent activity at $50 \mathrm{mg} / \mathrm{k}$.g.p.o. The experimental results are statistically significant at $\mathrm{P}<0.01$ level.
\end{abstract}

Keywords. Oxazolone scaffold; acetyl glycine; hypoglycemic activity; antiinflammatory.

\section{Introduction}

Diabetes mellitus (DM) is a major degenerative disease in the world today. ${ }^{1}$ Several epidemiological and clinical studies indicate a direct relationship between hyperglycemia and long-term complications such as retinopathy, nephropathy, neuropathy and angiopathy, etc. ${ }^{2,3}$ India has today become the diabetic capital of the world with over 20 million diabetics and this number is set to increase to 57 million by $2025 .{ }^{4} \mathrm{DM}$ is a multifactorial disease which is characterized by hyperglycemia, ${ }^{5}$ lipoprotein abnormalities, ${ }^{6}$ raised basal metabolic rate, ${ }^{7}$ defect in reactive oxygen species scavenging enzymes ${ }^{8}$ and high oxidative stress-induced damage to pancreatic beta cells. ${ }^{9}$ It is ranked seventh among the leading causes of death and is considered third when its fatal complications are taken into account. ${ }^{10} \mathrm{Sev}$ eral drugs such as sulfonylureas and biguanides are presently available to reduce hyperglycaemia in diabetes mellitus. These drugs have side effects and thus searching for a new class of compounds is essential to overcome this problems. ${ }^{11}$ Nevertheless, there is continuous search for alternative drugs; management of diabetes without any side effects is still a challenge to the medicinal chemist. Therefore, it is prudent to look for options in novel drugs for diabetes as well. Oxazole plays very vital role in the development of various biologically active agents such as analgesic, ${ }^{12}$ antiinflammatory, ${ }^{13}$ antidepressant, ${ }^{14}$ anticancer, ${ }^{15}$ antimicrobial, antidiabetic and antiobesity. ${ }^{16,17}$ Oxazol-5-ones contain

\footnotetext{
${ }^{*}$ For correspondence
}

numerous reactive sites allowing for a diverse set of possible modifications. This diverse reactivity makes them excellent substrates for their use in diversity oriented synthesis. ${ }^{18}$ The onset of insulin in body, which causes an abnormal effect on glucose metabolism, is related not only to the development of Type II diabetes but also to cardiovascular disease. ${ }^{19}$ Sulfonylureas, the first generation of antidiabetic agents such as chlorpropamide, tolbutamide and tolazamide are still in use but are less potent than the second generation drugs like glibenclamide, glipizide and glimepiride. Sulfonylureas are mostly subjected to hepatic metabolism, yielding less active or inactive metabolites that are then eliminated through the kidneys. Patients with impaired hepatic or renal function risk severe hypoglycemia because of accumulation of active drug in circulation. From the pioneering discovery of ciglitazone, ${ }^{20}$ a new class of thiazolidinedione based compounds has been developed to treat diabetic patients that can reverse the insulin resistance in non-insulin dependent diabetes mellitus (NIDDM). Amongst various substituted benzyl-2,4thiazolidinedione compounds, troglitazone,${ }^{21}$ pioglitazone, ${ }^{22}$ and rosiglitazone, ${ }^{23}$ are potentially antidiabetic compounds that have been clinically examined. The first marketed thiazolidinedione, troglitazone, ${ }^{24}$ was withdrawn because of increased risk of hepatotoxicity. ${ }^{25,26}$ The potent side effect i.e., hepatotoxicity limits the use of thiazolidine derivatives as safe drug candidates. In view of this, it was of considerable interest to synthesize the title compound with a hope to obtain potent biologically active and safe oral antidiabetic agents (figure 1). 


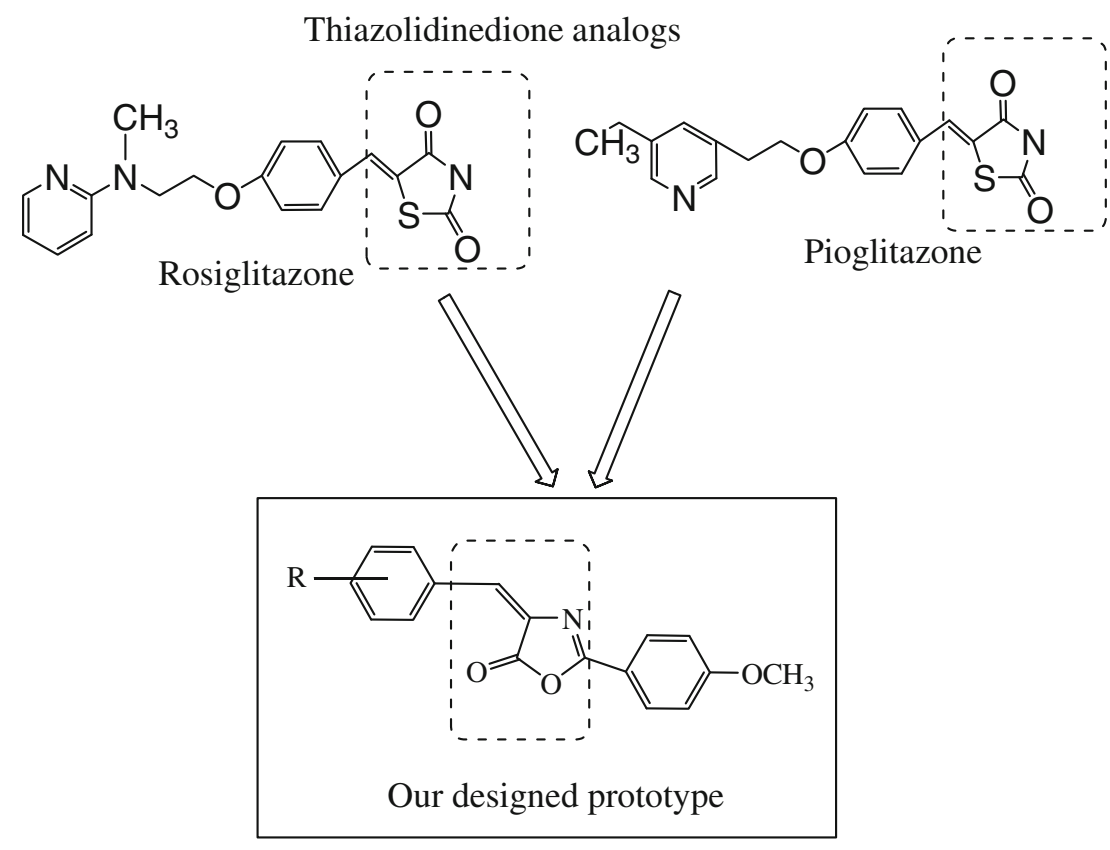

Figure 1. Structural resemblance of thiazolidinedione and oxazolone derivatives.

The starting material 4-methoxy benzoyl glycine was prepared by the reaction of glycine and 4-methoxy benzoyl chloride in the presence of sodium hydroxide. ${ }^{27}$ It was then reacted with substituted aromatic and hetero aldehyde in presence of acetic anhydride and glacial acetic acid to obtain oxazolones. The chemistry used to prepare these analogs is illustrated in scheme 1 . Their structures have been elucidated from UV-Vis, IR, ${ }^{1} \mathrm{H}$ NMR, Mass spectral data and elemental analysis. Physical data of synthesized compounds are given in table 1.

\section{Experimental}

\subsection{Material and methods}

All the chemicals were of synthetic grade and commercially procured from SD fine Chemicals Ltd. Mumbai, India. Melting points were recorded on a Buchi capillary melting point apparatus and are uncorrected. IR spectra were recorded on FT-IR8400S, Fourier Transform (SHIMADZU) Infrared spectrophotometer

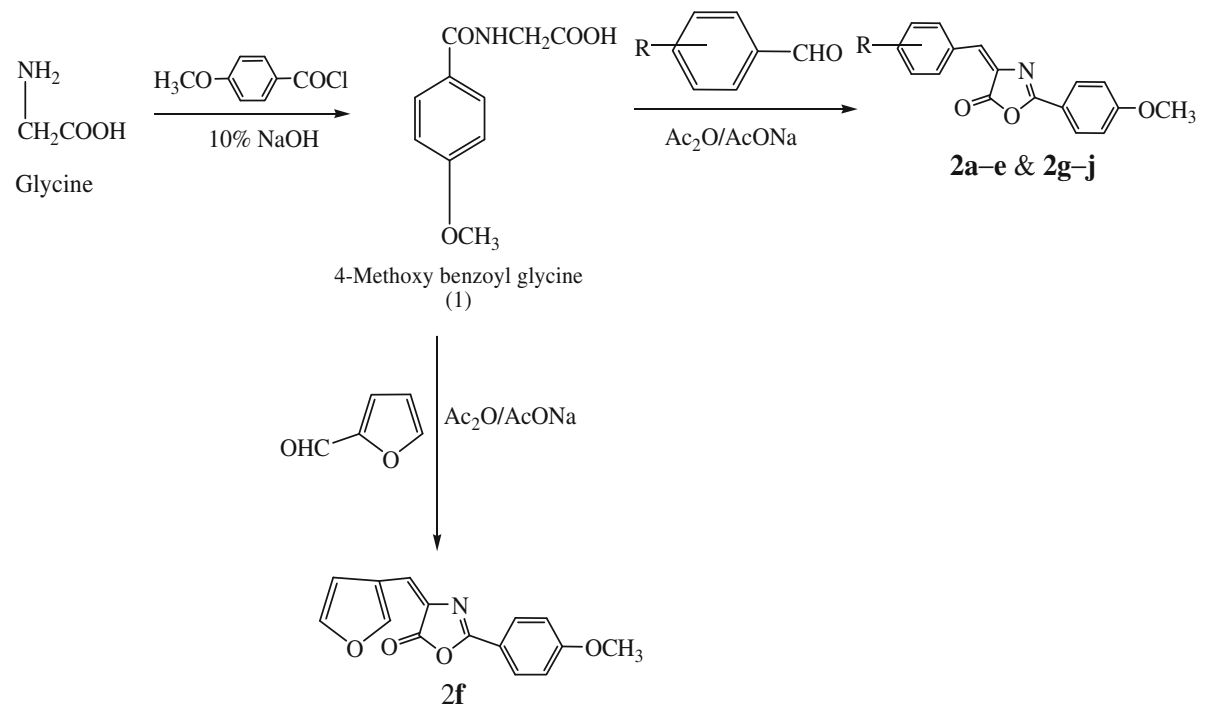

Scheme 1. Synthesis of oxazolone derivatives. 
Table 1. Physical data of synthesized compounds.

\begin{tabular}{lccccc}
\hline Compound & $\mathrm{R}$ & Mol. formula & Mol. weight & M.P $\left({ }^{\circ} \mathrm{C}\right)$ & Yield $(\%)$ \\
\hline $\mathbf{2 a}$ & $-\mathrm{H}$ & $\mathrm{C}_{17} \mathrm{H}_{13} \mathrm{NO}_{3}$ & 279 & $208-210$ & 65 \\
$\mathbf{2 b}$ & $2-\mathrm{Cl}$ & $\mathrm{C}_{17} \mathrm{H}_{12} \mathrm{ClNO}_{3}$ & 313 & $203-205$ & 68 \\
$\mathbf{2 c}$ & $4-\mathrm{OMe}$ & $\mathrm{C}_{18} \mathrm{H}_{15} \mathrm{NO}_{4}$ & 309 & $182-185$ & 60 \\
$\mathbf{2 d}$ & $3-\mathrm{OMe}$ & $\mathrm{C}_{18} \mathrm{H}_{15} \mathrm{NO}_{4}$ & 309 & $158-160$ & 71 \\
$\mathbf{2 e}$ & $3-\mathrm{Cl}$ & $\mathrm{C}_{17} \mathrm{H}_{12} \mathrm{ClNO}_{3}$ & 313 & $180-182$ & 69 \\
$\mathbf{2 f}$ & - & $\mathrm{C}_{15} \mathrm{H}_{11} \mathrm{NO}_{4}$ & 269 & $175-177$ & 60 \\
$\mathbf{2 g}$ & $2-\mathrm{OH}$ & $\mathrm{C}_{17} \mathrm{H}_{13} \mathrm{NO}_{4}$ & 295 & $170-172$ & 65 \\
$\mathbf{2 h}$ & phenyl ethenyl & $\mathrm{C}_{18} \mathrm{H}_{14} \mathrm{NO}_{3}$ & 292 & $168-170$ & 75 \\
$\mathbf{2 i}$ & $3-\mathrm{NO}_{2}$ & $\mathrm{C}_{17} \mathrm{H}_{12} \mathrm{~N}_{2} \mathrm{O}_{5}$ & 324 & $204-206$ & 72 \\
$\mathbf{2 j}$ & $4-\mathrm{N}-(\mathrm{Me})_{2}$ & $\mathrm{C}_{19} \mathrm{H}_{18} \mathrm{~N}_{2} \mathrm{O}_{3}$ & 322 & $206-208$ & 75
\end{tabular}

using $\mathrm{KBr}$ disc method. The ${ }^{1} \mathrm{H}-\mathrm{NMR}$ spectra were recorded in DMSO- $\delta 6$ on Perkin Elmer NMR Spectrophotometer-300 MHz. using TMS as an internal standard. Mass spectra were recorded on Micro mass Q-TOF and Shimadzu LCMS 2010A Mass spectrometer. Elemental analysis was performed at a Perkin Elmer Auto system XL Analyzer. Thin layer chromatography analyses were performed on pre-coated silica gel plates (G 350, Merck).

\subsection{Preparation of 4-methoxy benzoyl glycine}

Glycine $(0.1 \mathrm{~mol})$ was dissolved in $30 \mathrm{ml}$ sodium hydroxide $(2 \mathrm{~N})$ in a $500 \mathrm{ml}$ conical flask. The mixture was stirred vigorously with mechanical stirrer until the solid almost completely dissolved. 4-methoxy benzoyl chloride $(0.1 \mathrm{~mol})$ was added in five portions and stirred vigorously for further $1 \mathrm{~h}$. The crystallized 4-methoxy benzoyl glycine was left in refrigerator overnight. The crystals were filtered on a Buchner funnel, washed with ice cold water and dried at $100^{\circ} \mathrm{C}$. The melting point $145-149^{\circ} \mathrm{C}$, yield $80 \%$.

\subsection{General method for the preparation of 4-substituted-[Benzylidene]-2-(4-methoxy phenyl) oxazol-5-one $e^{28}(\mathbf{2 a - j})$}

A mixture of 4-methoxy benzoyl glycine $(0.01 \mathrm{~mol})$, substituted aromatic aldehyde $(0.02 \mathrm{~mol})$, anhydrous sodium acetate $(0.01 \mathrm{~mol})$ and acetic anhydride $(0.04 \mathrm{~mol})$ was refluxed for $1 \mathrm{~h}$ on a water bath with occasional stirring. The resulting mixture was left in refrigerator overnight. The solid thus obtained was filtered, washed with cold water, dried in hot air oven at $60^{\circ} \mathrm{C}$ and recrystallized from ethanol. All the title compounds were synthesized by adopting the same procedure with variation in reaction time. 2.3a 4-[Benzylidene]-2-(4-methoxy-phenyl) oxazol-5one (2a): Pale yellow powder; IR (KBr): $v\left(\mathrm{~cm}^{-1}\right)$, $1782(\mathrm{C}=\mathrm{O}), 1651(\mathrm{C}=\mathrm{N}), 1506(\mathrm{C}=\mathrm{C}-\mathrm{Ar})$, $3064(=\mathrm{C}-\mathrm{H}), 2914\left(\mathrm{CH}_{3}\right), 2762(\mathrm{Ar} \mathrm{C}-\mathrm{H}) ;{ }^{1} \mathrm{H}$ NMR $\left(300 \mathrm{MHz}, \mathrm{CDCl}_{3}, \delta \mathrm{ppm}\right): 3.90\left(\mathrm{~s}, 3 \mathrm{H},-\mathrm{OCH}_{3}\right), 7.37$ $(\mathrm{s}, \mathrm{H}, \mathrm{C}=\mathrm{C}-\mathrm{H}), 7.56(\mathrm{~d}, 2 \mathrm{H}, \mathrm{Ar}-\mathrm{H}), 7.19(\mathrm{~d}, 2 \mathrm{H}, \mathrm{Ar}-$ $\mathrm{H}), 8.09(\mathrm{t}, 1 \mathrm{H}, \mathrm{Ar}-\mathrm{H}) 8.18(\mathrm{~d}, 2 \mathrm{H}, \mathrm{Ar}-\mathrm{H}), 8.31(\mathrm{~d}, 2 \mathrm{H}$, $\mathrm{Ar}-\mathrm{H}$ ); MS (EI): $302 \mathrm{~m} / \mathrm{z}\left[\mathrm{M}+\mathrm{Na}^{+}\right]^{+}, 259,227,60$; Anal. Calcd for $\mathrm{C}_{17} \mathrm{H}_{13} \mathrm{NO}_{3}$ : C, 73.11; H, 4.69; N, 5.02; Found: C, 72.71; H, 4.19; N, 4.62.

$2.3 \mathrm{~b}$ 4-[2-Chloro-benzylidene]-2-(4-methoxy-phenyl)(4H)-oxazol-5-one $(\mathbf{2 b})$ : Yellow powder; IR $(\mathrm{KBr})$ $v\left(\mathrm{~cm}^{-1}\right): 1788(\mathrm{C}=\mathrm{O}), 1651(\mathrm{C}=\mathrm{N}), 1506(\mathrm{C}=$ $\mathrm{C}-\mathrm{Ar}), 3074(=\mathrm{CH}), 2922\left(\mathrm{CH}_{3}\right), 2833(\mathrm{Ar}-\mathrm{CH})$; ${ }^{1} \mathrm{H}$ NMR $\left(300 \mathrm{MHz}, \mathrm{CDCl}_{3} \delta \mathrm{ppm}\right): 3.81 \quad(\mathrm{~s}, 3 \mathrm{H}$, $\left.\mathrm{OCH}_{3}\right), 7.40-7.65(\mathrm{~s}, 1 \mathrm{H}, \mathrm{H}-\mathrm{C}=), 7.19$ (d, 2H, Ar$\mathrm{H}), 7.31(\mathrm{~d}, 2 \mathrm{H}, \mathrm{Ar}-\mathrm{H}), 7.44-7.56(\mathrm{~m}, 4 \mathrm{H}, \mathrm{Ar}-\mathrm{H})$; MS (EI): $311 \mathrm{~m} / \mathrm{z}[\mathrm{M}-2]^{+}, 283,255$; Anal. Calcd for $\mathrm{C}_{17} \mathrm{H}_{12} \mathrm{ClNO}_{3}$ : C, 65.08; H, 3.86; N, 4.46; Found: C, $65.50 ; \mathrm{H}, 3.36 ; \mathrm{N}, 4.86$.

$2.3 \mathrm{c}$ 4-[4-Methoxy-benzylidene]-2-(4-methoxy-phenyl) oxazol-5-one (2c): Deep yellow powder; IR $(\mathrm{KBr})$ $v\left(\mathrm{~cm}^{-1}\right): 1784(\mathrm{C}=\mathrm{O}), 1651(\mathrm{C}=\mathrm{N}), 1600(\mathrm{C}=$ $\mathrm{C}-\mathrm{Ar}), 3431(=\mathrm{C}-\mathrm{H}), 2928\left(\mathrm{CH}_{3}\right), 885,825(\mathrm{Ar}-\mathrm{CH})$; ${ }^{1} \mathrm{H}$ NMR $\left(300 \mathrm{MHz}, \mathrm{CDCl}_{3}, \delta \mathrm{ppm}\right): 3.32(\mathrm{~s}, 6 \mathrm{H}, 2 \times$ $\left.\mathrm{OCH}_{3}\right), 7.26$ (s, 1H, H-C=), 7.23 (d, 2H, Ar-H), 7.30 $(\mathrm{d}, 2 \mathrm{H}, \mathrm{Ar}-\mathrm{H}), 7.56(\mathrm{~d}, 2 \mathrm{H}, \mathrm{Ar}-\mathrm{H}), 8.38(\mathrm{~d}, 2 \mathrm{H}, \mathrm{Ar}-$ $\mathrm{H})$; MS (EI): $309 \mathrm{~m} / \mathrm{z}[\mathrm{M}]^{+}, 310,321,331,332[\mathrm{M}+$ $\left.\mathrm{Na}^{+}\right]^{+}$, 333; Anal. Calcd for $\mathrm{C}_{18} \mathrm{H}_{15} \mathrm{NO}_{4}: \mathrm{C}, 69.89 ; \mathrm{H}$, 4.89; N, 4.53; Found: C, 69.48; H,4.46; N, 4.91.

$2.3 \mathrm{~d}$ 4-[3-Methoxy-benzylidene]-2-(4-methoxy-phenyl) oxazol-5-one $(\mathbf{2 d})$ : Pale yellow powder; IR $(\mathrm{KBr}) v$ $\left(\mathrm{cm}^{-1}\right): 1784(\mathrm{C}=\mathrm{O}), 1656(\mathrm{C}=\mathrm{N}), 1604(\mathrm{C}=\mathrm{C}-$ $\mathrm{Ar}), 3074(=\mathrm{CH}), 2958\left(\mathrm{CH}_{3}\right), 883,835,771,678,604$ 
(Ar-CH); ${ }^{1} \mathrm{H}$ NMR $\left(300 \mathrm{MHz}, \mathrm{CDCl}_{3} \delta \mathrm{ppm}\right): 3.86$, $3.90\left(\mathrm{~s}, 6 \mathrm{H}, 2 \times \mathrm{OCH}_{3}\right), 7.45(\mathrm{~s}, 1 \mathrm{H}, \mathrm{H}-\mathrm{C}=), 7.10$ (d, 2H, Ar-H), 7.49 (d, 2H, Ar-H), 7.52-8.09 (m, 4H, $\mathrm{Ar}-\mathrm{H}) ; 310 \mathrm{~m} / \mathrm{z}[\mathrm{M}+1]^{+}, 331,332\left[\mathrm{M}+\mathrm{Na}^{+}\right]^{+}, 333$ Anal. Calcd for $\mathrm{C}_{18} \mathrm{H}_{15} \mathrm{NO}_{4}: \mathrm{C}, 69.89 ; \mathrm{H}, 4.89 ; \mathrm{N}, 4.53$; Found: C, 69.38; H,4.45; N, 4.94.

2.3e 4-[3-Chloro-benzylidene]-2-(4-methoxy-phenyl) oxazol-5-one (2e): Pale yellow powder; $\mathrm{IR}(\mathrm{KBr})$ $v\left(\mathrm{~cm}^{-1}\right): 1791(\mathrm{C}=\mathrm{O}), 1658(\mathrm{C}=\mathrm{N}), 1556(\mathrm{C}=$ $\mathrm{C}-\mathrm{Ar}), 2902\left(\mathrm{CH}_{3}\right), 3502(=\mathrm{C}-\mathrm{H}), 883,785,680,559$ $(\mathrm{Ar}-\mathrm{CH}) ;{ }^{1} \mathrm{H} \mathrm{NMR}\left(300 \mathrm{MHz}, \mathrm{CDCl}_{3}, \delta \mathrm{ppm}\right): 3.92$ (s, $\left.3 \mathrm{H},-\mathrm{OCH}_{3}\right), 7.57(\mathrm{~s}, 1 \mathrm{H}, \mathrm{H}-\mathrm{C}=), 7.23(\mathrm{~d}, 2 \mathrm{H}, \mathrm{Ar}-\mathrm{H})$, $7.96(\mathrm{~d}, 2 \mathrm{H}, \mathrm{Ar}-\mathrm{H}), 7.56(\mathrm{~d}, 2 \mathrm{H}, \mathrm{Ar}-\mathrm{H}), 8.01-8.38(\mathrm{~m}$, $4 \mathrm{H}, \mathrm{Ar}-\mathrm{H}) ; 313 \mathrm{~m} / \mathrm{z}\left[\mathrm{M}+\mathrm{Na}^{+}\right]^{+}, 336$ Anal. Calcd for $\mathrm{C}_{17} \mathrm{H}_{12} \mathrm{ClNO}_{3}: \mathrm{C}, 65.08 ; \mathrm{H}, 3.86$; N, 4.46; Found: $\mathrm{C}$, 65.51; H, 3.40; N, 4.96.

$2.3 \mathrm{f}$ 4-[Furan-3-ylmethylene]-2-(4-methoxy-phenyl)(4H)-oxazol-5-one (2f): Light green powder; IR $(\mathrm{KBr}) \cup\left(\mathrm{cm}^{-1}\right): 1790(\mathrm{C}=\mathrm{O}), 1654(\mathrm{C}=\mathrm{N}), 1556$ $(\mathrm{C}=\mathrm{C}-\mathrm{Ar}), 3601(=\mathrm{C}-\mathrm{H}), 3064\left(\mathrm{CH}_{3}\right), 2362$ [C$\mathrm{H}$ (furan)], 941, 860, 758, $696(\mathrm{Ar}-\mathrm{CH}) ;{ }^{1} \mathrm{H}$ NMR $\left(300 \mathrm{MHz}, \mathrm{CDCl}_{3,} \delta \mathrm{ppm}\right.$ ): 3.89 (s, $\left.3 \mathrm{H},-\mathrm{OCH}_{3}\right), 7.57$ (s, 1H, H-C=), $6.82(\mathrm{~d}, 2 \mathrm{H}, \mathrm{Ar}-\mathrm{H}), 6.83(\mathrm{~s}, \mathrm{H}, \mathrm{Ar}-$ H), 7.13 (d, 2H, Ar-H), 8.08 (d, 2H, Ar-H); $269 \mathrm{~m} / \mathrm{z}$ $[\mathrm{M}]^{+}, 292,\left[\mathrm{M}+\mathrm{Na}^{+}\right]^{+}, 227,142$; Anal. Calcd for $\mathrm{C}_{15} \mathrm{H}_{11} \mathrm{NO}_{4}$ : C, 66.91; H, 4.12; N, 5.20; Found: C, 67.41; H, 4.69; N, 5.57.

$2.3 \mathrm{~g}$ 4-[2-Hydroxy-benzylidene]-2-(4-methoxy-phenyl) oxazol-5-one $(2 \mathrm{~g})$ : Pale yellow crystal; IR $(\mathrm{KBr}) v$ $\left(\mathrm{cm}^{-1}\right): 1712(\mathrm{C}=\mathrm{O}), 1666(\mathrm{C}=\mathrm{N}), 1539(\mathrm{C}=$ $\mathrm{C}-\mathrm{Ar}), 3065(=\mathrm{C}-\mathrm{H}), 2837\left(\mathrm{CH}_{3}\right), 3007(\mathrm{C}-\mathrm{H}-\mathrm{Ar})$, 906, 848, 750, 601( $\mathrm{Ar}-\mathrm{CH})$; ${ }^{1} \mathrm{H}$ NMR $(300 \mathrm{MHz}$, $\left.\mathrm{CDCl}_{3}, \delta \mathrm{ppm}\right): 3.85\left(\mathrm{~s}, 3 \mathrm{H},-\mathrm{OCH}_{3}\right), 7.07(\mathrm{~s}, 1 \mathrm{H}, \mathrm{H}-$ $\mathrm{C}=), 7.94(\mathrm{~s}, 1 \mathrm{H}, \mathrm{OH}), 7.09$ (d, 2H, Ar-H), $7.22(\mathrm{~d}$, $2 \mathrm{H}, \mathrm{Ar}-\mathrm{H}), 7.24-7.97$ (m, 4H, Ar-H); $318 \mathrm{~m} / \mathrm{z}[\mathrm{M}+$ $\left.\mathrm{Na}^{+}\right]^{+}$Anal. Calcd for $\mathrm{C}_{17} \mathrm{H}_{13} \mathrm{NO}_{4}: \mathrm{C}, 69.15 ; \mathrm{H}, 4.44$; N, 4.74; Found: C, 69.56; H, 4.98; N, 4.52.

$2.3 \mathrm{~h}$ 4-[3-phenyl-allylidene]-2-(4-Methoxy-phenyl)(4H)-oxazol-5-one $(\mathbf{2 h})$ : Deep yellow powder; IR $(\mathrm{KBr}) \cup\left(\mathrm{cm}^{-1}\right): 1778(\mathrm{C}=\mathrm{O}), 1641(\mathrm{C}=\mathrm{N}), 1506$ $(\mathrm{C}=\mathrm{C}-\mathrm{Ar}), 3443(=\mathrm{C}-\mathrm{H}), 3103\left(\mathrm{CH}_{3}\right), 958,883,840$, $742(\mathrm{Ar}-\mathrm{CH}) ;{ }^{1} \mathrm{H}$ NMR $\left(300 \mathrm{MHz}, \mathrm{CDCl}_{3} \delta \mathrm{ppm}\right)$ : $3.88\left(\mathrm{~s}, 3 \mathrm{H},-\mathrm{OCH}_{3}\right), 7.14,7.18,7.28(3 \mathrm{H}$,olefinic pro- ton), $7.37(\mathrm{~d}, 2 \mathrm{H}, \mathrm{Ar}-\mathrm{H}), 7.48(\mathrm{~d}, 2 \mathrm{H}, \mathrm{Ar}-\mathrm{H}), 7.91(\mathrm{~d}$, $2 \mathrm{H}, \mathrm{Ar}-\mathrm{H}), 8.06(\mathrm{~d}, 2 \mathrm{H}, \mathrm{Ar}-\mathrm{H}) ; 315 \mathrm{~m} / \mathrm{z}\left[\mathrm{M}+\mathrm{Na}^{+}\right]^{+}$, 300,256; Anal. Calcd for $\mathrm{C}_{18} \mathrm{H}_{14} \mathrm{NO}_{3}: \mathrm{C}, 73.96 ; \mathrm{H}$, 4.83 ; N, 4.79; Found: C, 73.36; H, 4.33; N, 4.32.

$2.3 \mathrm{i}$ 4-[3-nitro-benzylidene]-2-(4-methoxy-phenyl)(4H)-oxazol-5-one (2i): Yellow powder; IR $(\mathrm{KBr}) v$ $\left(\mathrm{cm}^{-1}\right): 1790(\mathrm{C}=\mathrm{O}), 1654(\mathrm{C}=\mathrm{N}), 1606(\mathrm{C}=\mathrm{C}-$ $\mathrm{Ar}), 3103\left(\mathrm{CH}_{3}\right), 3443(=\mathrm{C}-\mathrm{H}), 2856(\mathrm{C}-\mathrm{H}-\mathrm{Ar}) ;{ }^{1} \mathrm{H}$ NMR (300 MHz, $\left.\mathrm{CDCl}_{3} \delta \mathrm{ppm}\right): 3.90(\mathrm{~s}, \mathrm{H}, \mathrm{OMe}), 7.41$ (s, 1H, H-C =), 7.20 (d, 2H, Ar-H ), 7.33 (d, 2H, Ar$\mathrm{H}), 7.80(\mathrm{~m}, 4 \mathrm{H}, \mathrm{Ar}-\mathrm{H})$; Anal. Calcd for $\mathrm{C}_{17} \mathrm{H}_{12} \mathrm{~N}_{2} \mathrm{O}_{5}$ : C, 62.96; H, $3.73 ; \mathrm{N}, 8.64 ; 347 \mathrm{~m} / \mathrm{z}[\mathrm{M}+\mathrm{Na}]^{+}$Found: C, $62.45 ; \mathrm{H}, 4.23 ; \mathrm{N}, 9.06$.

$2.3 \mathrm{j}$ 4-[4-Dimethylamino-benzylidene]-2-(4-methoxyphenyl)-(4H)-oxazol-5-one (2j): Orange powder; IR $(\mathrm{KBr}) v\left(\mathrm{~cm}^{-1}\right): 1780(\mathrm{C}=\mathrm{O}), 1647(\mathrm{C}=\mathrm{N}), 1604$ $(\mathrm{C}=\mathrm{C}-\mathrm{Ar}), 2976\left(\mathrm{CH}_{3}\right), 3443(=\mathrm{C}-\mathrm{H}), 2810(\mathrm{C}-\mathrm{H}-$ $\mathrm{Ar}) ;{ }^{1} \mathrm{H}$ NMR $\left(300 \mathrm{MHz}, \mathrm{CDCl}_{3} \delta \mathrm{ppm}\right): 3.88(\mathrm{~s}, 3 \mathrm{H}$, $\left.-\mathrm{OCH}_{3}\right), 3.07\left(\mathrm{~s}, 6 \mathrm{H}, \mathrm{N}-\mathrm{CH}_{3}\right), 7.37(1 \mathrm{H}, \mathrm{H}-\mathrm{C}=), 6.82$ (d, 2H, Ar-H), 6.97 (d, 2H, Ar-H), 7.01-8.17 (m, 4H, $\mathrm{Ar}-\mathrm{H}) ; 323 \mathrm{~m} / \mathrm{z}[\mathrm{M}+1]^{+}, 345\left[\mathrm{M}+\mathrm{Na}^{+}\right]^{+}$Anal. Calcd for $\mathrm{C}_{19} \mathrm{H}_{18} \mathrm{~N}_{2} \mathrm{O}_{3}$ : C, 70.79; H, 5.63; N, 8.64; Found: C, 70.38; H, 5.24; N, 8.22.

\subsection{Pharmacological evaluation}

2.4a Acute toxicity studies: Groups of six albino mice, weighing $20-25 \mathrm{~g}$ were fasted overnight and treated per orally with the test compounds. ${ }^{29,30}$ The dosage was varied from $100 \mathrm{mg} \mathrm{kg}^{-1}$ body weights. The animals were observed for $24 \mathrm{~h}$ for any signs of acute toxicity such as increased or decreased motor activity, tremors, convulsion, sedation, lacrimation, etc. No mortality of the animals was observed even after $24 \mathrm{~h}$. Hence the $\mathrm{LD}_{50}$ cut-off value of the test compounds was fixed as $100 \mathrm{mg} \mathrm{kg}^{-1}$, so that $100 \mathrm{mg} \mathrm{kg}^{-1}$ i.e., $1 / 10$ of cut-off value was taken as screening dose for evaluation of antidiabetic activity. All the animal experiments were conducted by the approval of Institutional Animal Ethics Committee, Himalayan Pharmacy Institute, East Sikkim, India. During the study period, guidelines of Committee for the Purpose of Control and Supervision of Experiments on Animals (CPCSEA), Institutional Animals Ethics Committee (IAEC) were followed for the maintenance of animals. The research work was approved by IAEC No: HPI/10/60/IAEC/0085. 


\subsection{Antidiabetic activity ${ }^{31,32}$}

2.5a Streptozotocin induction of experimental diabetes: The rats were injected intraperitoneally with streptozotocin dissolved in sterile normal saline at a dose of $60 \mathrm{mg} \mathrm{kg}^{-1}$ b.w. A blood glucose range of 200$300 \mathrm{mg}$ dL-1 was used for the experiment. Hyperglycemia was confirmed in animals after $72 \mathrm{~h}$ of streptozotocin injection.

2.5b Experimental design: Animals were divided into 12 groups of 6 animals $(n=6)$ : Group 1 diabetic animals (vehicle) received $0.5 \%$ CMC $(1 \mathrm{ml})$; Group 2 diabetic animals received rosiglitazone $100 \mathrm{mg} / \mathrm{kg}$. Groups (3-12) diabetic animals received compounds 2a-2j in a single dose of $50 \mathrm{mg} / \mathrm{kg}$ body weight per oral respectively for 7 days continuously.

2.5c Blood glucose measurement: Blood was withdrawn from the tail vain each time. Blood glucose was measured at $0,5^{\text {th }}$ and $10^{\text {th }}$ days interval. At the end of $0,5^{\text {th }}$ and $10^{\text {th }}$ day, blood sample was withdrawn from a tail vein by snipping the tip of the tail and the blood glucose level was measured by Accu Sure Blood Glucose Monitoring System (Dr. Gene Health \& Wellness).

2.5d Statistical analysis: Values are represented as mean \pm SEM. Data were analysed using analysis of variance and group means were compared with TukeyKramer Post ANOVA test. The values were considered when $\mathrm{P}<0.01$.

\section{Results and discussion}

All the compounds were screened in vivo for their oral hypoglycemic activity by streptozotocin-induced diabetic model in rat. All the compounds are having remarkable hypoglycemic property, however with a degree of variation. Blood glucose changes in treatment of diabetic rats with synthesized oxazolone derivatives were presented in (table 2). A significant increase in blood glucose was observed in diabetic rats. All the compounds $\mathbf{2 a}-\mathbf{2} \mathbf{j}$ had shown significant reduction in blood glucose as compared to control diabetic rats at $50 \mathrm{mg} / \mathrm{kg}$ body weight for $5^{\text {th }}$ and $10^{\text {th }}$ days. Rosiglitazone was taken as standard drug which showed $69.33 \%$ blood glucose lowering activity at the dose of $100 \mathrm{mg} / \mathrm{kg}$.p.o. Among all the derivatives, compound 2d $(64.36 \%)$ was found to be better hypoglycemic agent like the standard drug rosiglitazone $(100 \mathrm{mg} / \mathrm{kg} \mathrm{b.w})$ in reducing the blood glucose level. It is interesting to note that Compounds $\mathbf{2 c}(61.2 \%), \mathbf{2 d}(64.36 \%), \mathbf{2 j}$ (61.80\%), $2 \mathbf{i}(62.01 \%)$, and $2 \mathrm{e}(62.20 \%)$, having both electron donating or withdrawing substituent at meta and para position of the Oxazolone ring showed an appreciable increase in hypoglycemic activity at $50 \mathrm{mg} / \mathrm{kg}$, whereas electron withdrawing groups at ortho position as in $\mathbf{2 b}$ and $\mathbf{2 g}$ sustain the activity in STZ model but were found inferior. Surprisingly $\mathbf{2 f}(61.60 \%)$ has shown significant antihyperglycemic activity. Compound $\mathbf{2 h}$ (55.68\%), comparatively had shown poor hypoglycemic activity followed by compound $\mathbf{2 a}(62.80 \%)$.

Those compounds having electron donating substituent $\mathbf{2 c}$, 2d and $\mathbf{2 j}$ have shown $61.2 \%, 64.36 \%$ and $61.8 \%$ antihyperglycemic properties respectively,

Table 2. Hypoglycemic effects of synthesized test compounds of oxazolone derivatives.

\begin{tabular}{|c|c|c|c|c|}
\hline \multirow[t]{2}{*}{ Treatment (mg/kg b.w p.o) } & \multicolumn{4}{|c|}{ blood glucose level (mg/dl) } \\
\hline & 0-day & 5-day & 10-day & $\begin{array}{c}\% \text { anti hyperglycemic } \\
\text { activity }\end{array}$ \\
\hline Control (0.5\%CMC) & $338.8 \pm 6.5$ & $381.6 \pm 7.0^{* *}$ & $406.6 \pm 3.9^{* *}$ & \\
\hline Rosiglitazone100 & $339.5 \pm 4.4$ & $138.3 \pm 3.7^{* *}$ & $104.1 \pm 2.8^{* *}$ & 69.30 \\
\hline $\mathbf{2 a}$ & $343.6 \pm 5.8$ & $210.3 \pm 3.2^{* *}$ & $147.5 \pm 3.3^{* *}$ & 62.80 \\
\hline $2 \mathbf{b}$ & $342.1 \pm 4.2$ & $236.3 \pm 2.7^{* *}$ & $149.6 \pm 3.5^{* *}$ & 56.27 \\
\hline 2c & $338.3 \pm 4.8$ & $243.1 \pm 4.2^{* *}$ & $131 \pm 2.6^{* *}$ & 61.20 \\
\hline $2 d$ & $325 \pm 6.0$ & $195.5 \pm 2.2^{* *}$ & $115.8 \pm 2.1^{* *}$ & 64.36 \\
\hline $2 e$ & $352.3 \pm 5.1$ & $260.6 \pm 4.4^{* *}$ & $133 \pm 4.3^{* *}$ & 62.20 \\
\hline $2 f$ & $350.6 \pm 5.4$ & $256.5 \pm 6.8^{* *}$ & $134.67 \pm 3.0^{* *}$ & 61.60 \\
\hline $2 \mathrm{~g}$ & $356.6 \pm 6.1$ & $252.3 \pm 5.1^{* *}$ & $146.5 \pm 3.9^{* *}$ & 58.90 \\
\hline $2 \mathrm{~h}$ & $353.5 \pm 6.1$ & $259.3 \pm 5.1^{* *}$ & $156.67 \pm 5.7^{* *}$ & 55.68 \\
\hline $2 \mathbf{i}$ & $354.1 \pm 5.8$ & $249.5 \pm 4.1^{* *}$ & $134.5 \pm 4.8^{* *}$ & 62.01 \\
\hline $2 \mathbf{j}$ & $353.5 \pm 5.9$ & $257.1 \pm 5.4^{* *}$ & $134.83 \pm 4.2^{* *}$ & 61.80 \\
\hline
\end{tabular}

Values are expressed as mean \pm SEM; $(n=6), * * P<0.01$. 
where as the compounds attached with electron withdrawing substituent like $\mathbf{2 b}, \mathbf{2 e}$ and $\mathbf{2 i}$ show $56.27 \%$, $62.2 \%$ and $62.01 \%$ hypoglycemic property respectively. The compounds derived from cinnamaldehyde such as compounds $\mathbf{2 h}$ exhibited less $55.68 \%$ antidiabetic property and the compound $\mathbf{2 f}$ derived from furfural aldehyde exhibited $61.6 \%$ activity.

Amongst the compounds having electron donating groups, the meta substituted compound $\mathbf{2 d}$ has more activity than the para substituted compounds $\mathbf{2 c}$ and $\mathbf{2} \mathbf{j}$ comparing both para substituted compounds $\mathbf{2 c}$ and $\mathbf{2} \mathbf{j}$ are showing almost same activity. The electron withdrawing groups substituted at meta position $\mathbf{2 e}, \mathbf{2 i}$ are showing nearly same activity whereas ortho position $\mathbf{2 b}$ showing less activity. Interestingly, the compounds substituted with furan ring are also showing more activity which lies within the range of compounds $2 \mathbf{c}, \mathbf{2 e}, \mathbf{2 i}$ and $\mathbf{2 j}$.

The compound 2a that is unsubstituted by neither electron donating nor with electron withdrawing group shows significant activity (62.8\%). Amongst all the compounds, compound $\mathbf{2 h}$ exhibits very less activity. A conclusion can be arrived from these results that as compared to electron withdrawing groups; electron donating group enhanced the activity. The substituted electron donor and acceptor groups at ortho position decrease the activity. The compounds derived from aromatic and hetero aromatic aldehyde almost having equal activity. All the compounds activity is lower than the standard. Interestingly, compound $\mathbf{2 d}$ at $50 \mathrm{mg} / \mathrm{kg}$ lowers the blood glucose level almost equal to standard rosiglitazone.

Streptozotocin causes diabetes by the rapid depletion of $\beta$-cells and thereby brings about a reduction of insulin release. In our study, an increase in blood glucose level in diabetic rats confirmed the induction of diabetes mellitus. ${ }^{33}$ The oral administration of a single dose of synthesized compounds caused a significant reduction in blood glucose in diabetic rats. These results revealed that oxazolone derivatives may be effective in insulin-independent diabetes mellitus. The significant hypoglycemic effects of oxazolone derivatives in diabetic rats indicate that it can be mediated by stimulation of glucose utilization by peripheral tissues. Since the oxazolone derivatives are having structural resemblance with thiazolidinone with minor modification, it can be expected that the mode of action of the oxazolone derivatives may be similar to thiazolidinone derivatives. ${ }^{34}$ Hence oxazolone derivatives may act as agonist upon binding to peroxisome proliferatoractivated receptor (PPAR $\gamma$ ) which preferentially binds to DNA activating transcription of a wide variety of metabolic regulators. The regulators increase expression of a number of insulin responsive genes involved in the regulation of glucose and lipid metabolism. Studies have shown that there is a good correlation between PPAR $\gamma$-affinity and antihyperglycemic effects. ${ }^{35,36}$ It may increase glucose transport into muscles and adipose tissue by enhancing the synthesis and translocation of specific forms of the glucose transporter. However, it is expected that the synthesized compounds can act indirectly by stimulating the release of insulin into the bloodstream. From the observed result it is concluded that almost all the compounds $\mathbf{2} \mathbf{a}-\mathbf{2} \mathbf{j}$ reduced glucose level in diabetic rats. However, the effect of $\mathbf{2 d}$ is more pronounced in streptozotocin diabetic rats. Further toxicity studies and mode of action of hypoglycemic activity can provide an effective drug candidate for diabetes mellitus.

\section{Conclusion}

This study reports the synthesis and antidiabetic activity of novel oxazolones derivatives. The divergence in the antidiabetic activity of these compounds validates the significance of this study. The results of the study revealed that most of the compounds tested showed moderate to good antidiabetic activity. Structure and biological activity relationship of the title compounds showed the electron donating group enhanced the activity.

\section{Acknowledgements}

The authors are thankful to the Director Dr. H P Chhetri, Himalayan Pharmacy Institute, Majhitar, East Sikkim, India for providing the facilities to carry out the research work. The authors are also grateful to Dr. P K Kar, Department of Pharmacology, Himalayan Pharmacy Institute, who helped us to carry out the antidiabetic studies.

\section{References}

1. Ogbonnia S O, Odimegwu J I and Enwuru V N 2008 Afr. J. Biotechnol. 72535

2. Jr Porte D and Schwart M W 1996 Science 27699

3. Kristova V, Liskoya S, Sotnikova S, Vojtko R and Kurtansky A 2008 Physiol. Res. 5491

4. Sridhar G R 2000 Curr. Sci. 83791

5. Ugochukwu N H, Babady N E, Cobourne M and Gasset S R 2003 J. Biosci. 281

6. Scoppola A, Montecchi F R, Mezinger G and Lala A 2001 Atherosclerosis $\mathbf{1 5 6} 357$ 
7. Owu D U, Antai A B, Udofia K H, Obembe A O, Obasi K O and Eteng M U 2006 J. Biosci. 31575

8. Kesavulu M M, Giri R, Kameswara R B and Apparao C 2000 Diabetes Metabol. 26387

9. Nayeemunnisa A 2009 Int. J. Diabetes Metabol. 17105

10. Trivedi N A, Majumder B, Bhatt J D and Hemavathi K G 2004 Indian J. Pharmacol. 36373

11. Kamaeswara R B, Giri R, Kesavulu M M and Apparao C 2001 J. Ethnopharmacol. 7469

12. Lesieur and Aichaw H, Eur. Pat. 390 673/1990; 1991 Chem. Abstr. 114143

13. Ando K and Asai N, Eur. Pat. 664 385/1990

14. Decas P and Jarry C, Eur. Pat. 929 392/1990

15. Doctorovich V B, Burgess E M, Lambropoulos J, Lednicer D, Derveer D V and Zalkow L H 1994 J. Med. Chem. 37710

16. Pereira E R, Sanceleme M, Voldoire A and Prudhomme M 1997 Bioorg. Med. Chem. Lett. 72503

17. Viti G, Nannicini R, Ricci R, Pestellini V, Abelli L and Furio M 1994 Eur. J. Med. Chem. 29401

18. Jason F S, Robert M A and Jetze T J 2007 Chem. Soc. Rev. 361432

19. Olefsky J M, Garvey W T, Henry R R, Brillon D, Matthael S and Freidenberg G R 1998 Am. J. Med. 5A 86

20. Defronzo R A and Ann 2000 Intern. Med. 13373

21. Yoshioka T, Fujita T, Kanai T, Aizawa Y, Kuramada T, Hasegawa K and Horikoshi H 1989 J. Med. Chem. 32 421

22. Momose Y, Meguro K, Ikeda H, Hatanaka C, Oi S and Sohda T 1991 Chem. Pharm. Bull. 391440
23. Cantello B C C, Cawthorne M A, Haigh D, Hindley R M, Smith S A and Thurby P L 1994 Bioorg. Med. Chem. Lett. 41181

24. Krook A, Bjornholm M, Galuska D, Jiang X J, Fahlman R, Myers M G, Henriksson H W and Zierath J R 2000 Diabetes 49284

25. Fuchtenbusch M, Stand E and Schatz H 2000 Exp. Clin. Endocrinol. Diabetes $\mathbf{1 0 8} 151$

26. Scheen A J 2001 Drug Saf. 24873

27. Furniss B S, Hannaford A J, Smith P W G and Tatchell A R 1989 Vogel's textbook of practical organic chemistry (England: ELBS Longman) p. 907

28. Cantello B C C, Cawthorne M A, Haigh D, Hindley R M, Smith S A and Thurby P L 1994 Bioorg. Med. Chem. Lett. 41181

29. Litchfield J T and Wilcoxon E J 1949 J. Pharmacol. Exp. Ther. 9699

30. Raghavan P V 2000 Expert Consultant, CPCSEA, OECD, guideline no 420

31. Jarret R J, Keen H and Hardwick C 1970 Diabetes 19 724

32. Mackener C R, Saunders R N and Haettinger J R 1976 J. Toxi. Envi. Health 2139

33. Mohamed A K, Bierhaus A, Sciekofer S, Tritschler H, Ziegler H and Nawroth P P 1999 Biofactors 10175

34. Robert S and McDonald I M 2003 Burger's medicinal chemistry and drug discovery (New Jersey: John Wiley and Sons) pp. 28-30

35. Spiegelman B M 1998 Diabetes 47507

36. Staels B and Auwerx J 1997 Curr. Pharm. Des. 31 